\title{
A Novel Optimization Method for Bipolar Chaotic Toeplitz Measurement Matrix in Compressed Sensing
}

\author{
Rui Zhang $(\mathbb{D}$, Chen Meng, Cheng Wang $(\mathbb{D}$, and Qiang Wang \\ Shijiazhuang Campus, Army Engineering University, Shijiazhuang 050003, China \\ Correspondence should be addressed to Cheng Wang; 810134271@qq.com
}

Received 1 June 2021; Revised 27 June 2021; Accepted 14 July 2021; Published 31 July 2021

Academic Editor: Mu Zhou

Copyright (c) 2021 Rui Zhang et al. This is an open access article distributed under the Creative Commons Attribution License, which permits unrestricted use, distribution, and reproduction in any medium, provided the original work is properly cited.

\begin{abstract}
In this paper, a bipolar chaotic Toeplitz measurement matrix optimization algorithm for alternating optimization is presented. The construction of measurement matrices is one of the key techniques for compressive sensing from theory to engineering applications. Recent studies have shown that bipolar chaotic Toeplitz matrices, constructed by combining the intrinsic determinism of bipolar chaotic sequences with the advantages of Toeplitz matrices, have significant advantages over other measurement matrices in terms of memory overhead, computational complexity, and hard implementation. However, problems such as strong correlation and large interdependence coefficients between measurement matrices and sparse dictionaries may still exist in practical applications. To address this problem, we propose a new bipolar chaotic Toeplitz measurement matrix alternating optimization algorithm. Firstly, by introducing the structure matrix, the optimization problem of the measurement matrix is transformed into the optimization problem of the generating sequence, thus ensuring that the optimization process does not destroy the structural properties of the matrix; then, constraints are added to the values of the generating sequence during the optimization process, so that the optimized measurement matrix still maintains the bipolar properties. Finally, the effectiveness of the optimization algorithm in this paper is verified by simulation experiments. The experimental results show that the optimized bipolar chaotic Toeplitz measurement matrix can effectively reduce the reconstruction error and improve the reconstruction probability.
\end{abstract}

\section{Introduction}

Compressed sensing (CS) $[1,2]$ is a new framework for signal sampling. It enables to sample sparse signals at a sampling rate much lower than Nyquist's and to achieve accurate recovery of the original signal with high probability. The signal processing method based on compressed sensing theory does not depend on the bandwidth of the signal and can break the limitation of the sampling process by Nyquist's sampling theory. CS has broad application prospects in the fields of broadband signal acquisition [3], medical imaging $[4,5]$, and data compression [6], etc.

The measurement matrix design is one of the cores of compressed sensing theory [7]. On the one hand, the nature of the measurement matrix directly determines whether the compressive sampling process can fully retain the useful information of the original signal, and on the other hand, the design of the measurement matrix needs to take into account the implementation capability of the compressed sampling system $[8,9]$. Although the widely used random measurement matrices such as Gaussian and Bernoulli have good applicability, there are too many free elements in the matrices, and they are not conducive to hardware implementation. Based on the above two factors, the bipolar chaotic sequence is used to construct Toeplitz matrix as a measurement matrix for compressed sensing - called the bipolar chaotic Toeplitz measurement matrix-in the literature [10]. This measurement matrix is simple to generate and has few free elements, which greatly reduces the difficulty of hardware implementation and, at the same time, supports fast algorithms that can solve numerous problems related to convolutional operations. Although the restricted isometry property (RIP) of the bipolar Toeplitz measurement matrix was proved $[11,12]$, the constructed bipolar chaotic Toeplitz 
measurement matrix may still have a large correlation with the sparse dictionary during practical applications, which affects the compressed sampling reconstruction of the signal.

Measurement matrix optimization is an effective way to solve the above problem; the current optimization algorithms on the measurement matrix mainly focus on the optimization of random measurement matrix, such as the Elad algorithm [13], the Duarte-Carvajalino algorithm [14], and the Abolghasemi algorithm [15]. The strategy of alternating optimization between the matrix to be optimized and the target matrix in the Abolghasemi algorithm can effectively expand the search space and improve the optimization effect. Based on the idea of alternating optimization, a weighted measurement matrix optimization objective function was proposed in the literature [16] to improve the robustness of the compressive sampling system under the condition of considering both signal adaptation and the matrix's own characteristics. The literature [17] introduced the concept of parameter update in K-SVD to improve the measurement matrix update and improve the efficiency of matrix optimization. The literature [18] proposed a new joint optimization algorithm of the measurement matrix and sparse dictionary to improve the signal compression-aware reconstruction effect by constructing a new objective function. However, the above alternating optimization algorithm does not consider the possible structural constraints of the measurement matrix itself, so the structural properties of the measurement matrix itself will be destroyed after optimization. On the premise of considering the structural properties of the matrix, the literature [19] proposed an alternating optimization algorithm for sparse measurement matrices, and the literature [20] proposed an alternating optimization algorithm for cyclic matrices, but both algorithms are not applicable to the bipolar Toeplitz measurement matrix.

From the above analysis, it can be seen that the existing measurement matrix optimization methods do not meet the optimization needs of the bipolar Toeplitz measurement matrix. The reasons for this are mainly the following two aspects. On the one hand, the bipolar Toeplitz measurement matrix has a special matrix structure, and the existing measurement matrix optimization algorithms cannot guarantee that the matrix structure remains unchanged after optimization. On the other hand, the matrix elements of the bipolar Toeplitz measurement matrix have only two values, and the existing measurement matrix optimization algorithm will destroy the bipolar characteristics of the matrix elements.

To address the above problems, a new bipolar chaotic Toeplitz measurement matrix alternation optimization algorithm is proposed in this paper. In this paper, starting from the structural characteristics of Toeplitz matrix, the Toeplitz matrix is decomposed into the form of weighted summation of multiple structural matrices, thus converting the matrix optimization problem into the optimization problem of matrix generation sequence, which ensures that the original structure of the matrix optimization process remains unchanged. Second, a threshold function is introduced to constrain the values of the generated sequence during the iterative process, which ensures that the optimized matrix still maintains the bipolar property. The experimental results show that the optimized bipolar chaotic Toeplitz measurement matrix compression-aware reconstruction error is reduced and the reconstruction probability is significantly improved.

\section{Description of the Problem}

2.1. Bipolar Chaotic Sequence. Before constructing a bipolar chaotic Toeplitz measurement matrix, a bipolar sequence needs to be constructed first. The pseudorandom sequences based on chaotic systems have deterministic generating functions and good statistical independence, which are more conducive to hardware implementation, and for this reason, a bipolar sequence generation method based on chaotic systems is used in this paper.

A logistic chaotic system is a commonly used method to generate chaotic sequences. Considering the problem of generating bipolar Toeplitz measurement matrix, the following mapping function for the logistic chaotic system is used in [21]:

$$
x_{j+1}=\mu\left(1-2 x_{j}^{2}\right), j \in \mathbb{N}
$$

where $x_{j} \in[-1,1]$ and $\mu \in[0,1]$; this function is more suitable for the modulation of digital signals. When $\mu \geqslant$ 0.8371 , the logistic chaotic system has a positive Lyapunov exponent and the system enters a chaotic state; when $\mu=1$, the mapping is traversed on the interval $[-1,1]$. As can be seen from Figure 1, the time series goes through three different evolutionary stages of unstable immobility $\longrightarrow$ cycle $\longrightarrow$ chaos in turn. The closer $\mu$ is to 1 , the closer the range of values of $x$ distributed over the entire region of -1 to 1 ; this means that the more obvious the chaos is. When the logistic mapping is extremely sensitive to changes in the initial value after the value of $\mu$ is determined, the structure of the entire chaotic system is very different when a small change in the initial value occurs.

The invariant probability density function of the logistic chaotic sequence is

$$
\rho(x)=\left\{\begin{array}{l}
\frac{1}{\pi \sqrt{1-x^{2}}}, x \in[-1,1] \\
0, \text { else. }
\end{array}\right.
$$

Then, the mean value of the series is

$$
\bar{x}=\lim _{J \longrightarrow \infty} \frac{1}{J} \sum_{j=1}^{J} x_{j}=\int_{-1}^{1} x \rho(x) d x=0 .
$$

By repeated iterations of Equation (1), a set of logistic real-valued chaotic sequences $\left\{x_{j}\right\}_{j=0}^{\infty}$ can be generated. 


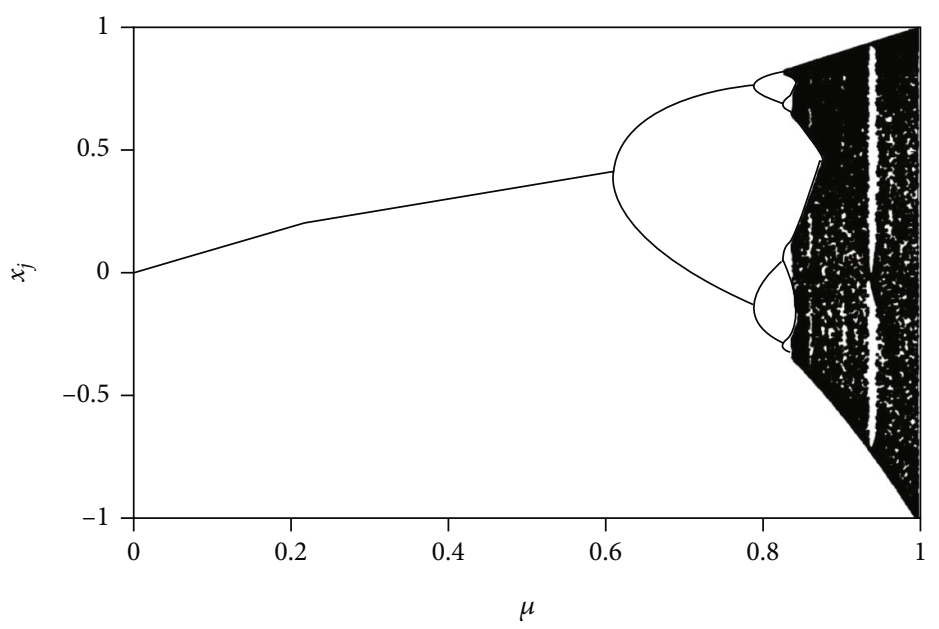

Figure 1: Logistic map bifurcation diagram.

Subsequently, a bipolar chaotic threshold function is introduced for this chaotic sequence $\left\{x_{j}\right\}_{j=0}^{\infty}$, which is

$$
A_{j}=T\left(x_{j}\right)= \begin{cases}+1, & x_{j} \geq 0, \\ -1, & x_{j}<0 .\end{cases}
$$

Then, the sequence $A_{j}$ constitutes a set of bipolar chaotic sequences. When $A_{j+1}$ obtains \pm 1 , the value interval of $x_{j}$ is taken as follows, respectively:

$$
A_{j+1}=\left\{\begin{array}{l}
+1, \quad x_{j} \in\left(-\frac{1}{\sqrt{2}}, \frac{1}{\sqrt{2}}\right) \\
-1, \quad x_{j} \in\left(-1,-\frac{1}{\sqrt{2}}\right) \cup\left(\frac{1}{\sqrt{2}}, 1\right) .
\end{array}\right.
$$

Then, using Equation (2), we can get the equal probability of $A_{j+1}$ obtaining \pm 1 , both being 0.5 .

2.2. Bipolar Chaotic Toeplitz Measurement Matrix. To ensure the applicability of the bipolar Toeplitz matrix alternating optimization algorithm in this paper, two Toeplitz measurement matrices are constructed using the bipolar chaotic sequence described above [10].

$$
\Phi_{1} \in \mathbb{R}^{M \times N}=\frac{1}{\sqrt{M}}\left(\begin{array}{ccccc}
A_{N} & A_{N-1} & \cdots & A_{2} & A_{1} \\
A_{N+1} & A_{N} & \cdots & A_{3} & A_{2} \\
\vdots & \vdots & \ddots & \vdots & \vdots \\
A_{M+N-1} & A_{M+N-2} & \cdots & A_{M+1} & A_{M}
\end{array}\right)
$$

and $\boldsymbol{\Phi}_{2} \in \mathbb{R}^{M \times N}=\left[\boldsymbol{\Phi}^{(1)} \boldsymbol{\Phi}^{(2)} \cdots \boldsymbol{\Phi}^{(b)}\right]$, where

$$
\begin{gathered}
\Phi^{(i)}(i=1,2, \cdots, b) \in \mathbb{R}^{M \times N / b} \\
{\left[\begin{array}{ccccc}
A_{N}^{(i)} & A_{N-1}^{(i)} & \cdots & A_{2}^{(i)} & A_{1}^{(i)} \\
A_{N+1}^{(i)} & A_{N}^{(i)} & \cdots & A_{3}^{(i)} & A_{2}^{(i)} \\
\vdots & \vdots & \ddots & \vdots & \vdots \\
A_{M+N-1}^{(i)} & A_{M+N-2}^{(i)} & \cdots & A_{M+1}^{(i)} & A_{M}^{(i)}
\end{array}\right] .}
\end{gathered}
$$

The scalar $1 / \sqrt{M}$ in Equation (6) is used to normalize the columns of $\boldsymbol{\Phi}$ to ensure that the energy of the original signal $x$ is consistent with the energy of the measured sample signal $y$ during the dimensionality reduction $\mathbb{R}^{N} \longrightarrow \mathbb{R}^{M} . \Phi_{2}$ is the multiblock Toeplitz measurement matrix, and when $b=1$, the multiblock Toeplitz measurement matrix degenerates to the conventional single-block Toeplitz measurement matrix. Since the generating sequences of both measurement matrices are bipolar chaotic sequences, the two measurement matrices are collectively referred to as bipolar chaotic Toeplitz measurement matrices in this paper.

The RIP properties of the bipolar chaotic Toeplitz measurement matrix have been effectively demonstrated in [9] and are not repeated here. The focus of this paper is on the optimization of the bipolar chaotic Toeplitz measurement matrix. Although the bipolar chaotic Toeplitz measurement matrix constructed using Equation (6) can satisfy the RIP property with high probability, there may still be problems such as strong correlation between the measurement matrix and the sparse dictionary and large interdependence coefficients during practical applications, so it is necessary to optimize the constructed bipolar chaotic Toeplitz measurement matrix to further improve the performance of the measurement matrix.

Currently, the core idea of measurement matrix optimization is to reduce the interdependence coefficients of the measurement matrix by reducing the values of the 
nondiagonal elements of the Gram matrix. And the main methods to reduce the values of nondiagonal elements are threshold shrinkage, gradient descent, and singular value decomposition. However, the above methods all destroy the structural properties of the measurement matrix and the bipolar properties of the matrix elements, so they are not suitable for the optimization of bipolar chaotic Toeplitz measurement matrices. Based on this, this paper presents a new bipolar chaotic Toeplitz measurement matrix alternation optimization algorithm.

\section{Bipolar Chaotic Toeplitz Matrix Alternating Optimization Algorithm}

3.1. Optimization of the Objective Function. For a onedimensional signal $x \in \mathbb{R}^{N}$, the compressed perception process can be expressed as

$$
y=\Phi x=\Phi \Psi s
$$

where $y \in \mathbb{R}^{M}$ is the measurement data, $\Phi$ is the measurement matrix, $\Psi$ is the sparse dictionary, $s$ is the sparse vector, and when the signal $x$ is sparse, $\Psi$ is the identity matrix.

The first attempt to consider the optimal design of measurement matrix was made in [12]. It states that by reducing the correlation between the measurement matrix and the sparse dictionary, the compression-aware reconstruction of the signal can be effectively improved. We define the Gram matrix:

$$
\mathbf{G}=\Psi^{T} \boldsymbol{\Phi}^{T} \boldsymbol{\Phi} \boldsymbol{\Psi}
$$

The measurement matrix objective function can be expressed as follows:

$$
\min _{\mathbf{G}, \mathbf{G}_{\text {ideal }}}\left\|\mathbf{G}-\mathbf{G}_{\text {ideal }}\right\|_{F}^{2} \text {. }
$$

The bipolar chaotic Toeplitz matrix used in this paper has obvious structural properties. In order to ensure that the measurement matrix optimization process still maintains its original structural properties, the measurement matrix is decomposed as follows:

$$
\boldsymbol{\Phi}=\sum_{j=1}^{J} A_{j} \boldsymbol{\Phi}^{j},
$$

where $J$ is the number of free elements in the measurement matrix, and $J$ in $\Phi$ takes the value of $M+N-1$, and $\Phi^{j}$ is the structure matrix corresponding to element $A_{j}$. The matrix consists of 0 and $1 / \sqrt{M}$ elements and has the same matrix dimension as $\Phi$. If the matrix element in $a$ is $1 / \sqrt{M}$, it means that the matrix element in that position in $\Phi$ is $A_{j}$. So $\Phi$ can be expressed as

$$
\begin{aligned}
\boldsymbol{\Phi}_{1}=\left(\begin{array}{ccccc}
A_{N} & A_{N-1} & \cdots & A_{2} & A_{1} \\
A_{N+1} & A_{N} & \cdots & A_{3} & A_{2} \\
\vdots & \vdots & \ddots & \vdots & \vdots \\
A_{M+N-1} & A_{M+N-2} & \cdots & A_{M+1} & A_{M}
\end{array}\right) \\
=\left(\begin{array}{ccc}
0 & \cdots & A_{1} \\
\vdots & \ddots & \vdots \\
0 & \cdots & 0 \\
0 & \cdots & 0
\end{array}\right)+\cdots+\left(\begin{array}{ccc}
\vdots & \ddots & \vdots \\
A_{M+N-1} & \cdots & 0
\end{array}\right) \\
=A_{1} \boldsymbol{\Phi}^{1}+\cdots+A_{M+N-1} \boldsymbol{\Phi}^{M+N-1},
\end{aligned}
$$

that is,

$\boldsymbol{\Phi}^{1}=\left(\begin{array}{ccc}0 & \cdots & \frac{1}{\sqrt{M}} \\ \vdots & \ddots & \vdots \\ 0 & \cdots & 0\end{array}\right), \cdots, \Phi^{M+N-1}=\left(\begin{array}{ccc}0 & \cdots & 0 \\ \vdots & \ddots & \vdots \\ \frac{1}{\sqrt{M}} & \cdots & 0\end{array}\right)$.

Similarly, in $\Phi_{2}$,

$$
\boldsymbol{\Phi}_{2}=A_{1} \boldsymbol{\Phi}^{1}+\cdots+A_{b M+N-b} \boldsymbol{\Phi}^{b M+N-b}
$$

where

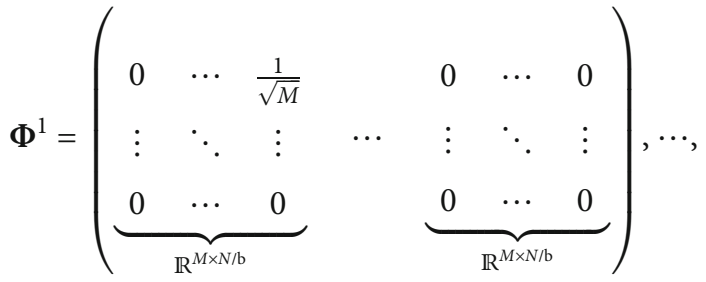

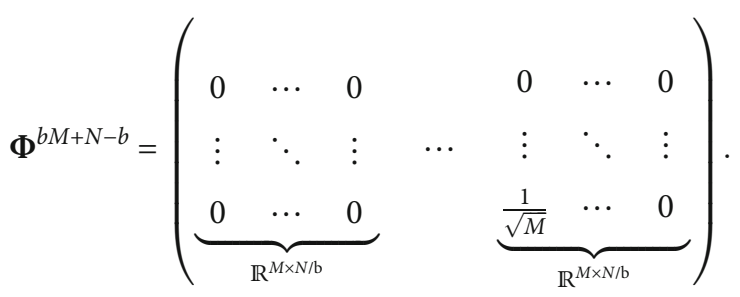

Although $\Phi_{1}$ and $\Phi_{2}$ have different structures, they can both be expressed in the form of Equation (11) and, given a value of $b$, the measurement matrix is determined by the generating sequence $A_{j}$ only. To ensure that the structural properties of the measurement matrix are not destroyed by the optimization process, the measurement matrix optimization objective function is further expressed as 
$\min _{\left\{A_{j}\right\}, \mathbf{G}_{\text {ideal }}} f\left(\left\{A_{j}\right\}, \mathbf{G}_{\text {ideal }}\right)=\left\|\boldsymbol{\Psi}^{T}\left(\sum_{j=1}^{J} A_{j}\left(\boldsymbol{\Phi}^{j}\right)^{T}\right)\left(\sum_{j=1}^{J} A_{j} \boldsymbol{\Phi}^{j}\right) \boldsymbol{\Psi}-\mathbf{G}_{\text {ideal }}\right\|_{F}^{2}$.

This ensures that the structure matrix remains unchanged during the optimization process and only the sequence $A_{j}$ is optimized.

3.2. The Proposed Method. For the multiparameter optimization problem in Equation (16), the following alternating optimization strategy is used in this paper:

(i) Fixed the latest $\left\{A_{j}\right\}$, updated $G_{\text {ideal }}$

(ii) Fixed the latest $G_{\text {ideal }}$, updated $\left\{A_{j}\right\}$

$G_{\text {ideal }}$ is updated using the following contraction operation for a fixed $\left\{A_{j}\right\}$ :

$\mathbf{G}_{\text {ideal }}^{i+1}\left(n, n^{\prime}\right)=\left\{\begin{array}{l}1, \quad n=n^{\prime}, \\ \operatorname{sign}\left(\mathbf{G}^{i}\right) \min \left(\left|\mathbf{G}^{i}\left(n, n^{\prime}\right)\right|, \eta\right), \quad n \neq n^{\prime},\end{array}\right.$

where $i$ is the number of iterations, $\mathbf{G}^{i}=\Psi^{T}\left(\sum_{j=1}^{J} A_{j}^{i}\right.$ $\left.\left(\boldsymbol{\Phi}^{j}\right)^{T}\right)\left(\sum_{j=1}^{J} A_{j}^{i} \boldsymbol{\Phi}^{j}\right) \boldsymbol{\Psi}$, and sign (.) is the sign function, $\eta=$ $\sqrt{(M-N) /(N(M-1))}$.

The elements in the sequence $\left\{A_{j}\right\}$ are updated one by one using the gradient descent algorithm under the condition that the $G_{\text {ideal }}$ is fixed:

$$
A_{j}^{i+1}=A_{j}^{i}-\beta \nabla f_{A_{j}^{i}}\left(A_{j}^{i}, \mathbf{G}_{\text {ideal }}^{i}\right),
$$

where $\beta$ is the gradient descent step and $\nabla f_{A_{j}^{i}}(\cdot)$ is the gradient operator. In this paper, the expression for $\nabla f_{A_{j}^{i}}\left(A_{j}^{i}, \mathbf{G}_{\text {ideal }}^{i}\right)$ is derived as follows:

$$
\begin{gathered}
f\left(\left\{A_{j}\right\}, \mathbf{G}_{\text {ideal }}\right)= \\
\left\|\boldsymbol{\Psi}^{T}\left(\sum_{j=1}^{J} A_{j}\left(\boldsymbol{\Phi}^{j}\right)^{T}\right)\left(\sum_{j=1}^{J} A_{j} \boldsymbol{\Phi}^{j}\right) \boldsymbol{\Psi}-\mathbf{G}_{\text {ideal }}\right\|_{F}^{2},
\end{gathered}
$$

let

$$
\begin{gathered}
a=\left(\boldsymbol{\Phi}^{j} \boldsymbol{\Psi}\right)^{T} \boldsymbol{\Phi}^{j} \boldsymbol{\Psi} \\
b=\left(\boldsymbol{\Phi}^{j} \boldsymbol{\Psi}\right)^{T} \sum_{j}^{\prime \neq j} J A_{j}{ }^{\prime} \boldsymbol{\Phi}^{j^{\prime}} \boldsymbol{\Psi}+\sum_{j}^{\prime \neq j} J A_{j}{ }^{\prime}\left(\boldsymbol{\Phi}^{j^{\prime}} \boldsymbol{\Psi}\right)^{T} \boldsymbol{\Phi}^{j} \boldsymbol{\Psi}, \\
c=\sum_{j}{ }^{\prime \neq j} J A_{j}{ }^{\prime}\left(\boldsymbol{\Phi}^{j^{\prime}} \boldsymbol{\Psi}\right)^{T} \sum_{j}{ }^{\prime \neq j} J A_{j}^{\prime} \boldsymbol{\Phi}^{j^{\prime}} \boldsymbol{\Psi}-\mathbf{G}_{\text {ideal }} .
\end{gathered}
$$

Then Equation (19) can be simplified as

$$
f\left(\left\{A_{j}\right\}, \mathbf{G}_{\text {ideal }}\right)=\left\|A_{j}^{2} a+A_{j} b+c\right\|_{F}^{2} .
$$

$\nabla f_{A_{j}^{i}}\left(A_{j}^{i}, \mathbf{G}_{\text {ideal }}^{i}\right)$ can be calculated by the following equation:

$$
\begin{gathered}
\nabla f_{A_{j}^{i}}\left(A_{j}^{i}, \mathbf{G}_{\text {ideal }}^{i}\right)= \\
\left(4 A_{j}^{i} \operatorname{vec}^{T}(a)+2 \operatorname{vec}^{T}(b)\right) \operatorname{vec}\left(\mathbf{G}^{i}-\mathbf{G}_{\text {ideal }}^{i}\right),
\end{gathered}
$$

where vec $(\cdot)$ is the matrix vectorization operator. The derivation process converts the $F$-parametrization of the matrix to the 2-parametrization of the vector by the $\operatorname{vec}(\cdot)$ operator, which gives the result in Equation (22).

3.3. Constraints for Generating Sequences. The introduction of the structure matrix $\Phi^{j}$ ensures that the structural properties of the measurement matrix are not destroyed during the alternating optimization process, but it is not guaranteed that the elements of the optimized matrix maintain their original bipolar properties. For this reason, this paper introduces a bipolar threshold function in sequence construction to constrain the sequence $\left\{A_{j}\right\}$ during the iterative process from the perspective of bipolar chaotic sequence construction:

$$
A_{j}^{i+1}= \begin{cases}+1, & A_{j}^{i+1} \geq 0 \\ -1, & A_{j}^{i+1}<0\end{cases}
$$

Then, by combining Equation (18) and Equation (23), the bipolar properties of the matrix elements will be effectively preserved during the optimization process. When the iterations converge, the optimized measurement matrix can be given by (11). At this point, the alternating optimization algorithm for the bipolar chaotic Toeplitz matrix in this paper is shown in Algorithm 1.

\section{Experiments and Analysis}

In order to verify the effectiveness of the bipolar chaotic Toeplitz measurement matrix alternating optimization algorithm in this paper, numerical simulation experiments are conducted to analyse the optimization effect of the measurement matrix and the compression-aware reconstruction effect of the optimized matrix.

4.1. Optimization Performance Analysis. The bipolar chaotic sequence is constructed using the logistic mapping and the bipolar threshold function, and then, the single block Toeplitz measurement matrix $\Phi_{1}$ and the multiblock Toeplitz measurement matrix $\Phi_{2}$ are constructed according to Equations (6) and (7), respectively. The parameters of the measurement matrix are set as follows: $N=256, M=128, b=2$. Setting the sparse dictionary as an identity matrix and the maximum number of iterations as 30 , the optimization effect of the two measurement matrices is shown in Figure 2. 
Input: Measurement matrix $\Phi$, sparse dictionary $\Psi$

Output: Optimized measurement matrix $\boldsymbol{\Phi}_{\text {opt }}$

Step 1: Using the input measurement matrix $\boldsymbol{\Phi}$, construct the sequence $\left\{A_{j}\right\}$ as well as the structure matrix $\left\{\boldsymbol{\Phi}^{j}\right\}$.

Step 2: Complete the update of $G_{\text {ideal }}$ by Equation (15)

Step 3: Using Equation (18), complete the update of sequence element $A_{j}$ one by one

Step 4: Constrain the updated element $A_{j}$ using Equation (23)

Step 5: Determine if the iteration termination condition is met, if so, go to Step 6, otherwise, go back to Step 2.

Step 6: Output optimized measurement matrix $\boldsymbol{\Phi}_{\text {opt }}$ using Equation (11)

Algorithm 1: The process of the proposed optimization method.

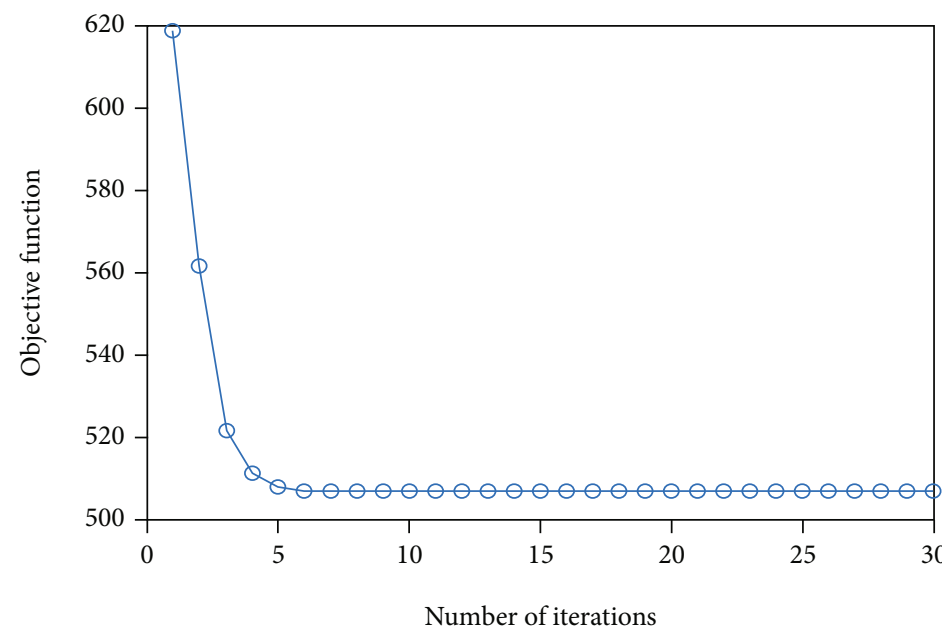

(a)

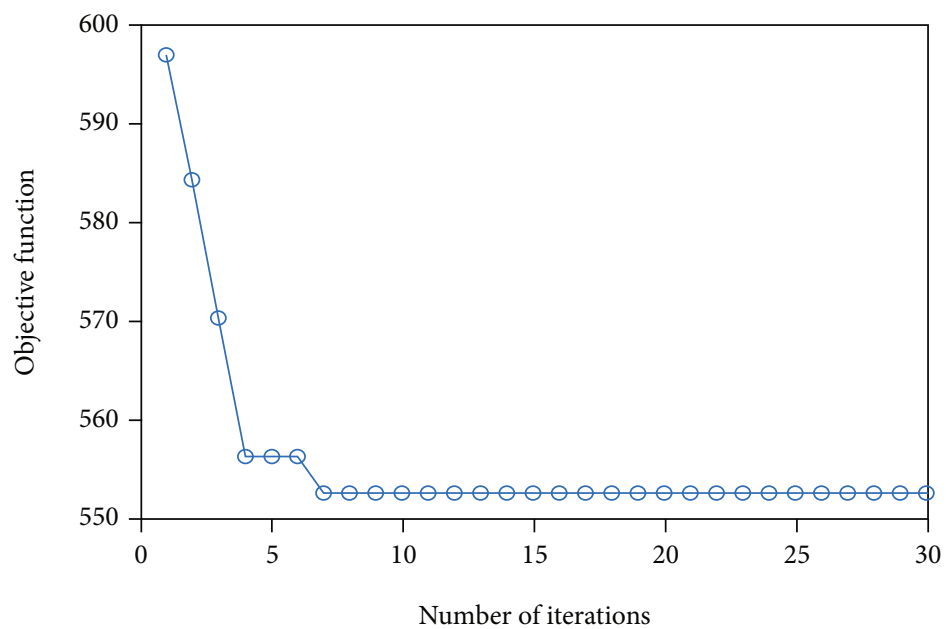

(b)

FIGURE 2: Optimization performance: (a) single-block bipolar chaotic Toeplitz measurement matrix and (b) multiblock bipolar chaotic Toeplitz measurement matrix.

As can be seen from the Figure 2, the optimization objective function decreases with the increase in the number of iterations and finally converges to stability. Compared with other measurement matrices, the structural properties of the bipolar chaotic Toeplitz measurement matrix and the bipolar nature of the matrix elements largely limit the
TABLE 1: Correlation coefficients.

\begin{tabular}{lcccc}
\hline & \multicolumn{2}{c}{ Original } & \multicolumn{2}{c}{ Optimized } \\
& $\mu_{\max }$ & $\mu_{\mathrm{av}}$ & $\mu_{\max }$ & $\mu_{\mathrm{av}}$ \\
\hline $\boldsymbol{\Phi}_{1}$ & 0.4587 & 0.0965 & 0.3437 & 0.0662 \\
$\boldsymbol{\Phi}_{2}$ & 0.5781 & 0.1001 & 0.3594 & 0.0724 \\
\hline
\end{tabular}




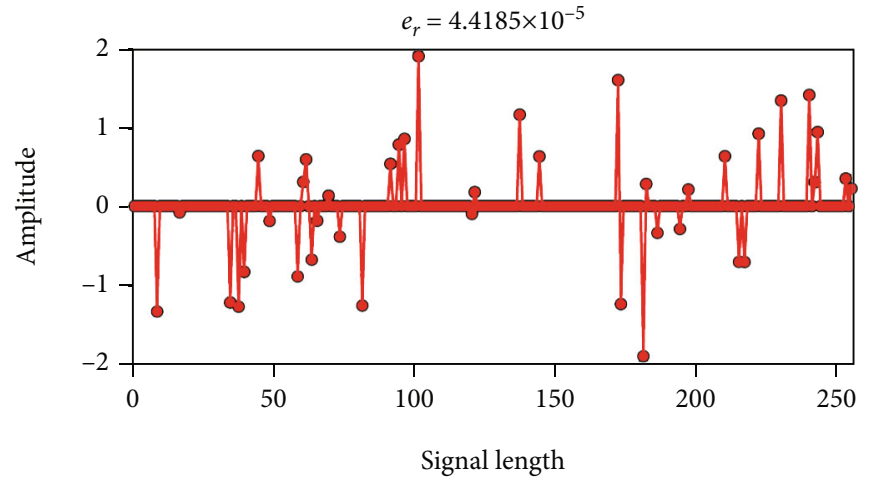

(a)

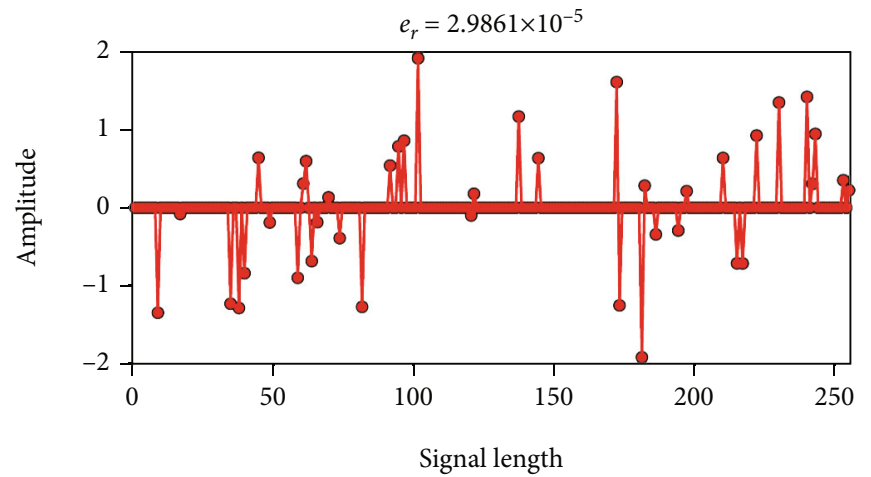

(c)

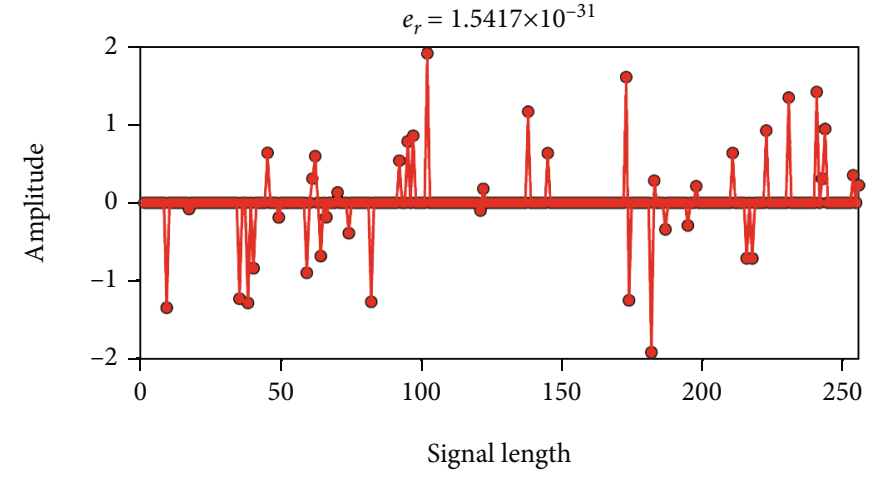

(b)

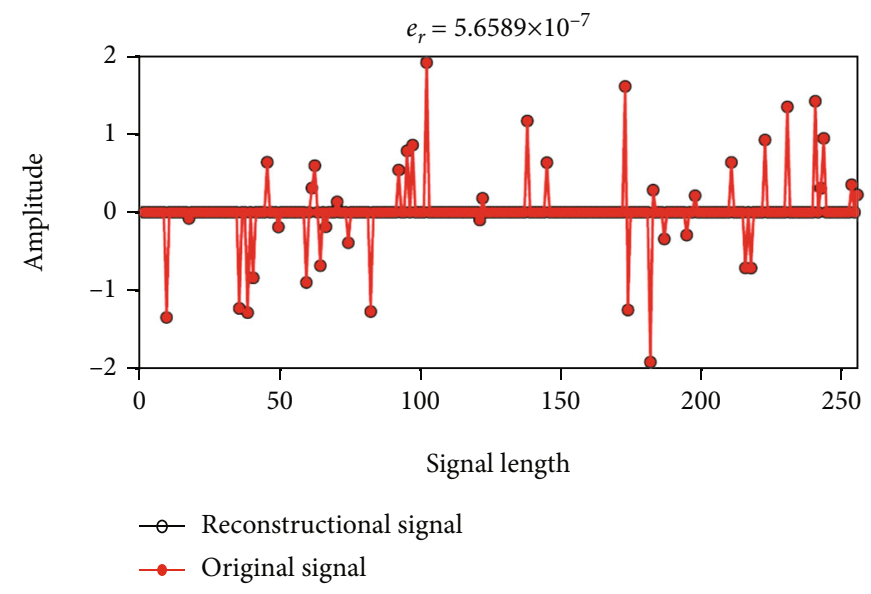

(d)

Figure 3: Reconstruction performance: (a) single-block bipolar chaotic Toeplitz measurement matrix, (b) optimized single-block bipolar chaotic Toeplitz measurement matrix, (c) multiblock bipolar chaotic Toeplitz measurement matrix, and (d) optimized multiblock bipolar chaotic Toeplitz measurement matrix.

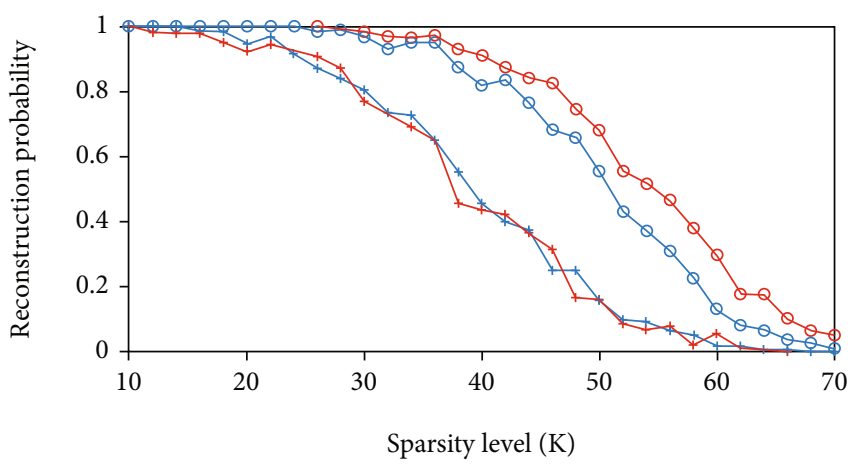

(a)

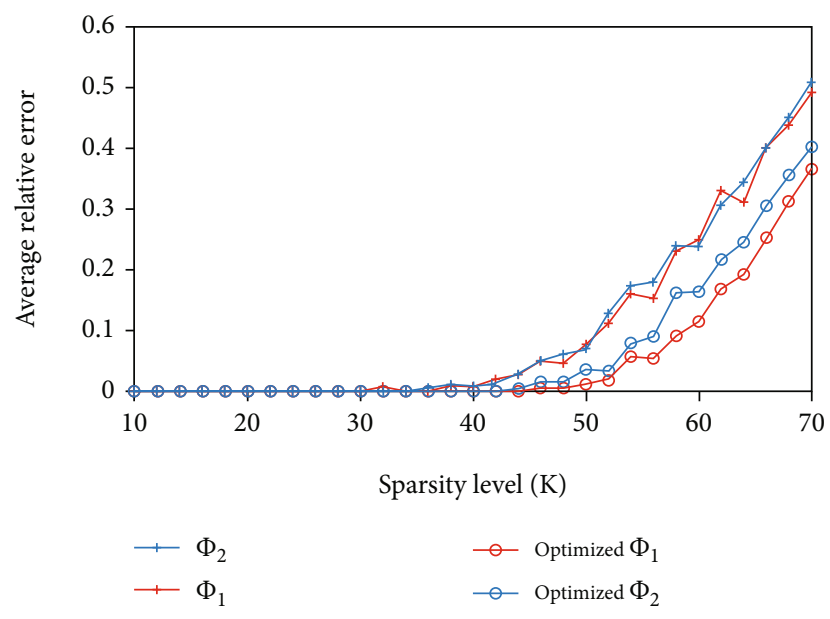

(b)

FIGURE 4: Reconstruction performance with different $K$ : (a) reconstruction probability and (b) average relative error. 


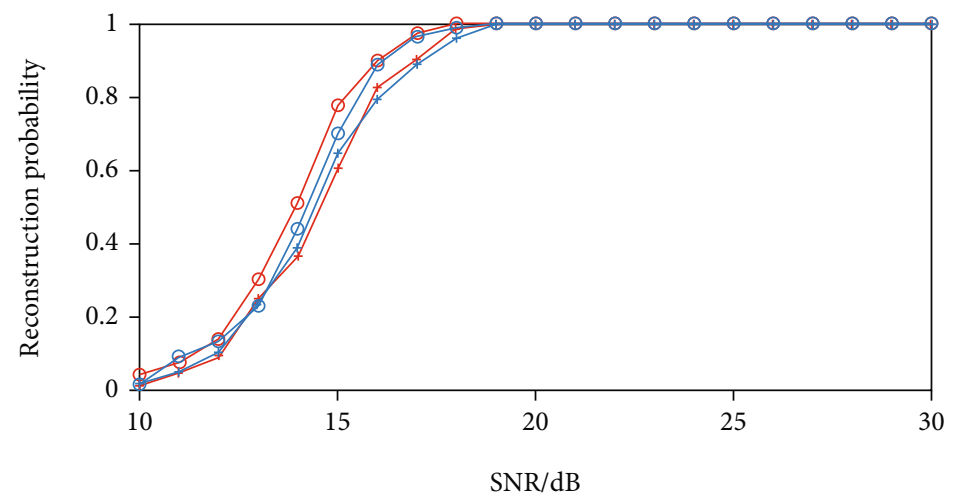

(a)

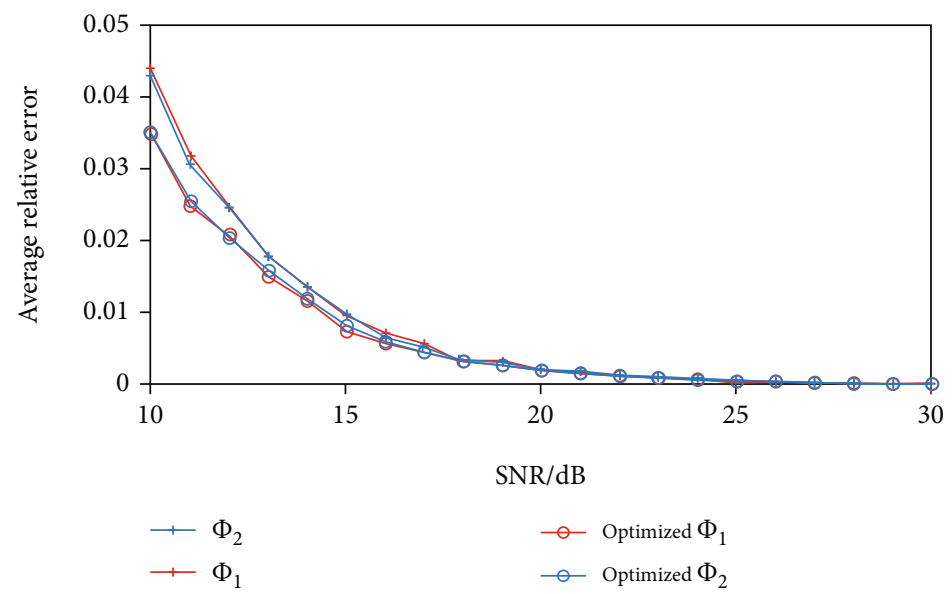

(b)

FIGURE 5: Reconstruction performance with different SNRs: (a) reconstruction probability and (b) average relative error.

optimizable space of the measurement matrix, so the iterative convergence of this matrix is faster. After optimization, the correlation coefficients of the measurement matrix are shown in Table 1 , where

$$
\begin{gathered}
\mu_{\max }=\max _{1 \leq n, n^{\prime} \leq N, n \neq n^{\prime}}\left|\mathbf{G}\left(n, n^{\prime}\right)\right|, \\
\mu_{\mathrm{av}}=\frac{1}{N(N-1)} \sum_{1 \leq n, n^{\prime} \leq N, n \neq n^{\prime}}\left|\mathbf{G}\left(n, n^{\prime}\right)\right| .
\end{gathered}
$$

As can be seen in Table 1, the optimized measurement matrix correlation coefficients $\mu_{\max }$ as well as $\mu_{\mathrm{av}}$ have been significantly reduced.

4.2. Analysis of the Effect of One-Dimensional Signal Reconstruction. The optimized measurement matrix was used to carry out simulation experiments on the compressive-perceptual reconstruction of one-dimensional signals and to analyse the effect of the optimized measurement matrix on the compressive-perceptual reconstruction effect of one-dimensional signals. During the simulation, a time-domain sparse signal was used for the onedimensional signal, at which time the sparse dictionary was an identity matrix. Setting the sparsity $K=30$, the effect of compressive-aware reconstruction for four different measurement matrices (single bipolar chaotic Toeplitz measurement matrix, optimized single bipolar chaotic Toeplitz measurement matrix, multiblock bipolar chaotic Toeplitz measurement matrix, and optimized multiblock bipolar chaotic Toeplitz measurement matrix) is shown in Figure 3. The reconstruction algorithm uses an orthogonal matching tracking algorithm [22], and to further quantify the reconstruction effect, the relative error is introduced as follows:

$$
e_{r}=\frac{\|\widehat{\boldsymbol{x}}-\boldsymbol{x}\|_{2}^{2}}{\|\boldsymbol{x}\|_{2}^{2}} \text {. }
$$

From the reconstruction results, it can be seen that all four measurement matrices achieve an effective reconstruction of the original signal at $M=128$, but a comparison of the reconstruction relative error shows that the optimized single/multiblock bipolar chaotic Toeplitz measurement matrix has significantly lower values of reconstruction relative error than the unoptimized single/multiblock bipolar chaotic Toeplitz measurement matrix. 


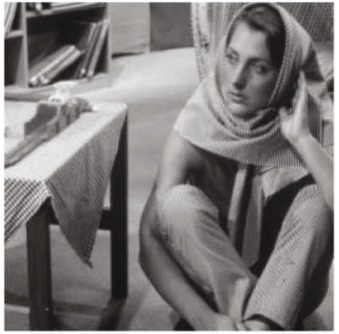

(a)

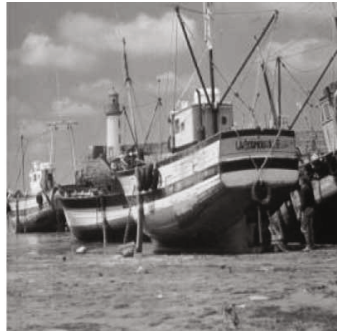

(b)

Figure 6: Original images: (a) "Barbara" and (b) "Boat."

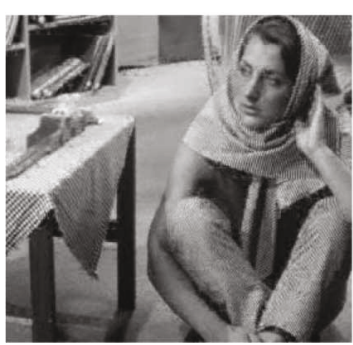

(a)

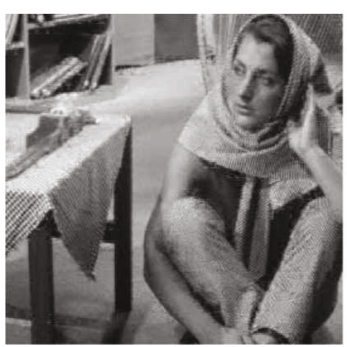

(b)

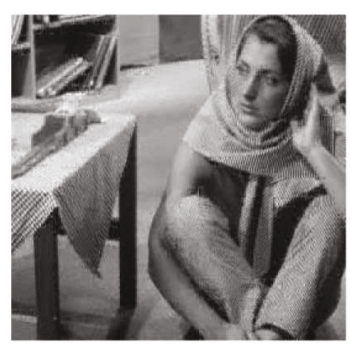

(c)

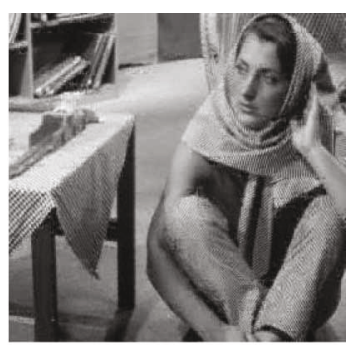

(d)

FIGURE 7: Reconstructed "Barbara": (a) single-block bipolar chaotic Toeplitz measurement matrix, $e_{r}=2.3077 \times 10^{-4}$; (b) optimized singleblock bipolar chaotic Toeplitz measurement matrix, $e_{r}=1.4181 \times 10^{-4}$; (c) multiblock bipolar chaotic Toeplitz measurement matrix, $e_{r}=$ $2.0132 \times 10^{-4}$; and (d) optimized multiblock bipolar chaotic Toeplitz measurement matrix, $e_{r}=1.8460 \times 10^{-4}$.

A different sparsity $K$ is set, and the reconstruction effect of the measurement matrix on different sparse signals is analysed. In the experiment, the position and amplitude of the sparse signals are generated randomly. 1000 Monte Carlo experiments were conducted under the same experimental conditions, and the reconstruction was successful when the relative error of reconstruction was lower than $10^{-4}$; otherwise, the reconstruction failed. The reconstruction probabilities of the four measurement matrices under different conditions of sparsity $K$ and the average relative errors are shown in Figure 4, where the average relative error is the average of 1000 Monte Carlo experiments.

As can be seen in Figure 4, the increasing sparsity leads to a decreasing reconstruction probability and an increasing reconstruction error. The optimized measurement matrices are more suitable for one-dimensional signals with different sparse locations and amplitudes, as the correlation coefficients $\mu_{\max }$ and $\mu_{\mathrm{av}}$ are significantly reduced. At this point, it can be seen from Figure 4 that the reconstruction probabilities of the optimized $\boldsymbol{\Phi}_{1}$ and $\boldsymbol{\Phi}_{2}$ are higher than those of the unoptimized $\Phi_{1}$ and $\boldsymbol{\Phi}_{2}$ for the same sparsity, while the average relative errors are significantly lower than those of the unoptimized $\boldsymbol{\Phi}_{1}$ and $\boldsymbol{\Phi}_{2}$.

In practical applications, the signal measurement process will inevitably be noisy, so the noise of different signal-tonoise ratios (SNR) is added to the compressed sampling process to analyse the reconstruction effect of the optimized bipolar chaotic Toeplitz measurement matrix under noisy conditions. In the experiments, the sparsity was set to 12 , and the reconstruction probability of the four measurement matrices without noise was close to 1 . Under the noisy condition, the reconstruction was successful when the reconstruction relative error was below $10^{-2}$; otherwise, the reconstruction failed. The reconstruction probabilities and the average relative errors of the four measurement matrices with different SNRs are shown in Figure 5.

4.3. Analysis of the Effect of Two-Dimensional Image Reconstruction. The optimization of the bipolar chaotic Toeplitz measurement matrix was further validated using twodimensional images. The two natural images ("Barbara" and "Boat") shown in Figure 6 were chosen as the experimental objects, both with a dimension of $256 \times 256$. The above image was divided into 256 image blocks which were $16 \times$ 16 for a compressed perceptual reconstruction experiment. The sparse dictionary used in the experiments is an orthogonal cosine dictionary. Setting the sampling rate to 0.5 , the reconstructed images after compressive sampling with different measurement matrices are shown in Figures 7 and 8.

As the images are not ideally sparse signals under the orthogonal cosine dictionary, there is local blurring in the reconstructed images after compressive sampling compared to the original images. Further comparison of the reconstruction errors shows that the optimized single/multiblock bipolar chaotic Toeplitz measurement matrix has a lower relative reconstruction error than the unoptimized single/multiblock bipolar Toeplitz measurement matrix. Thus, after optimization, the bipolar chaotic Toeplitz measurement matrix is effectively improved for compression-aware reconstruction in $2 \mathrm{D}$ images. 


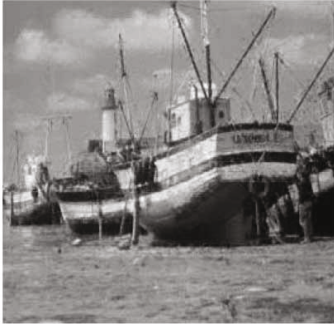

(a)

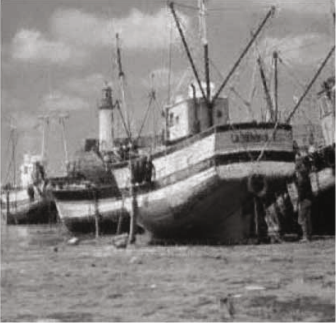

(b)

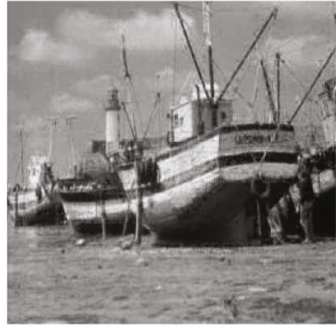

(c)

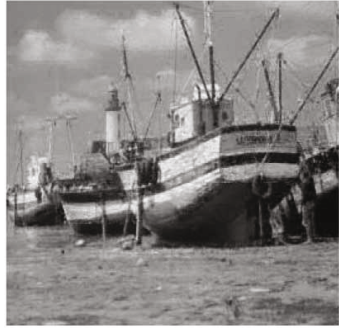

(d)

FIGURE 8: Reconstructed "Boat": (a) single-block bipolar chaotic Toeplitz measurement matrix, $e_{r}=3.172 \times 10^{-4}$; (b) optimized single-block bipolar chaotic Toeplitz measurement matrix, $e_{r}=1.7814 \times 10^{-4}$; (c) multiblock bipolar chaotic Toeplitz measurement matrix, $e_{r}=2.8183$ $\times 10^{-4}$; and (d) optimized multiblock bipolar chaotic Toeplitz measurement matrix, $e_{r}=2.4414 \times 10^{-4}$.

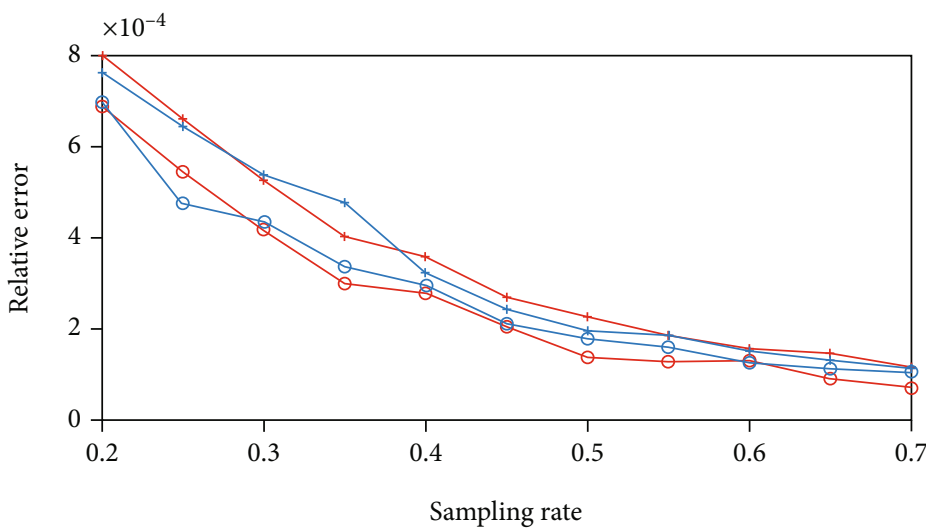

(a)

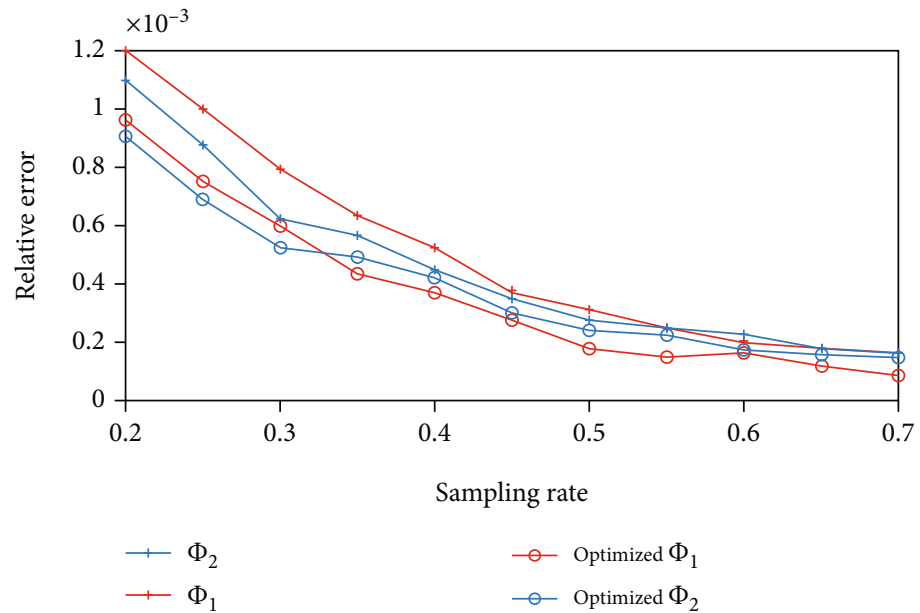

(b)

FIGURE 9: Reconstruction error with different sampling rates: (a) "Barbara" and (b) "Boat."

To further analyse the effect of sampling rate on the effect of image compression-aware reconstruction, the sampling rate was set to increase from 0.2 to 0.7 , and the relative error after compression-aware reconstruction of the four measurement matrices was analysed at different sampling rates, and the experimental results are shown in Figure 9. In the figure, as the sampling rate increases, the number of sampling points keeps increasing; therefore, the relative error of compressed sensing reconstruction keeps decreasing. And comparing the four measurement matrices, it can be seen that at the same sampling rate, the optimized single/multiblock bipolar chaotic Toeplitz measurement matrix has a lower relative reconstruction error than the unoptimized single/multiblock bipolar chaotic Toeplitz measurement matrix. This experimental result shows that the alternating optimization algorithm of the bipolar chaotic Toeplitz measurement matrix in this paper can effectively improve the compression-aware reconstruction performance of the measurement matrix at different sampling rates. 


\section{Conclusions}

In this paper, a bipolar chaotic Toeplitz measurement matrix optimization algorithm with alternating optimization is proposed to address the problem that existing measurement matrix optimization algorithms are not applicable in bipolar chaotic Toeplitz measurement matrices. The algorithm in this paper ensures that the structural properties of the optimized measurement matrix remain unchanged by introducing a structure matrix and then ensures that the bipolar properties of the optimized matrix elements remain unchanged by introducing constraints. The experimental results show that the optimization process effectively reduces the correlation coefficient of the measurement matrix, and the reconstruction error is effectively reduced, and the reconstruction probability is significantly improved when the optimized measurement matrix is applied to compressed perceptual reconstruction of $1 \mathrm{D}$ signals and $2 \mathrm{D}$ images.

\section{Data Availability}

The data used to support the findings of this study are available from the corresponding author upon request.

\section{Conflicts of Interest}

The authors declare that they have no conflicts of interest.

\section{Acknowledgments}

This work was funded by the National Natural Science Foundation of China (Grant No. 61501493).

\section{References}

[1] D. L. Donoho, "Compressed sensing," IEEE Transactions on Information Theory, vol. 52, no. 4, pp. 1289-1306, 2006.

[2] E. J. Candes, J. Romberg, and T. Tao, "Robust uncertainty principles: exact signal reconstruction from highly incomplete frequency information," IEEE Transactions on Information Theory, vol. 52, no. 2, pp. 489-509, 2006.

[3] H. Zhao, L. Qiao, J. Zhang, and N. Fu, "Generalized random demodulator associated with fractional Fourier transform," Circuits Systems \& Signal Processing, vol. 37, no. 11, pp. 5161-5173, 2018.

[4] M. Ambrosanio, P. Kosmas, and V. Pascazio, "A multithreshold iterative DBIM-based algorithm for the imaging of heterogeneous breast tissues," IEEE Transactions on Biomedical Engineering, vol. 66, no. 2, pp. 509-520, 2019.

[5] C. Dai, H. Che, and M. F. Leung, "A neurodynamic optimization approach for L1 minimization with application to compressed image reconstruction," International Journal on Artificial Intelligence Tools, vol. 30, no. 1, article 2140007, 2021.

[6] Q. Wang, C. Meng, W. Ma, C. Wang, and L. Yu, "Compressive sensing reconstruction for vibration signals based on the improved fast iterative shrinkage-thresholding algorithm," Measurement, vol. 142, pp. 68-78, 2019.

[7] Z. Wei, J. Zhang, Z. Xu, Y. Liu, and K. Okarma, "Measurement matrix optimization via mutual coherence minimization for compressively sensed signals reconstruction," Mathematical
Problems in Engineering, vol. 2020, Article ID 7979606, 18 pages, 2020.

[8] R. Arie, A. Brand, and S. Engelberg, "Compressive sensing and sub-Nyquist sampling," IEEE Instrumentation and Measurement Magazine, vol. 23, no. 2, pp. 94-101, 2020.

[9] T. Ragheb, J. N. Laska, H. Nejati, S. Kirolos, and Y. Massoud, "A prototype hardware for random demodulation based compressive analog-to-digital conversion," in 2008 51st Midwest Symposium on Circuits and Systems, pp. 37-40, Knoxville, TN, USA, 2008.

[10] H. Gan, S. Xiao, T. Zhang, and F. Liu, "Bipolar measurement matrix using chaotic sequence," Communications in Nonlinear Science and Numerical Simulation, vol. 72, pp. 139-151, 2019.

[11] L. Yu, J. P. Barbot, G. Zheng, and H. Sun, “Toeplitz-structured chaotic sensing matrix for compressive sensing," in 2010 7th International Symposium on Communication Systems, Networks \& Digital Signal Processing (CSNDSP 2010), pp. 229233, Newcastle Upon Tyne, UK, 2010.

[12] B. Zhang, J. Zhou, and J. Li, "Improved shrinkage estimators of covariance matrices with Toeplitz-structured targets in small sample scenarios," IEEE Access, vol. 7, no. 99, pp. 116785116798, 2019.

[13] M. Elad, "Optimized projections for compressed sensing," IEEE Transactions on Signal Processing, vol. 55, no. 12, pp. 5695-5702, 2007.

[14] J. M. Duarte-Carvajalino and G. Sapiro, "Learning to sense sparse signals: simultaneous sensing matrix and sparsifying dictionary optimization," IEEE Transactions on Image Processing, vol. 18, no. 7, pp. 1395-1408, 2009.

[15] V. Abolghasemi, S. Ferdowsi, and S. Sanei, “A gradient-based alternating minimization approach for optimization of the measurement matrix in compressive sensing," Signal Processing, vol. 92, no. 4, pp. 999-1009, 2012.

[16] H. Bai, G. Li, S. Li, Q. Li, Q. Jiang, and L. Chang, "Alternating optimization of sensing matrix and sparsifying dictionary for compressed sensing," IEEE Transactions on Signal Processing, vol. 63, no. 6, pp. 1581-1594, 2015.

[17] T. Hong and Z. Zhu, "An efficient method for robust projection matrix design,” Signal Processing, vol. 143, pp. 200-210, 2018.

[18] H. Bai and X. Li, "Measurement-driven framework with simultaneous sensing matrix and dictionary optimization for compressed sensing," IEEE Access, vol. 8, no. 99, pp. 3595035963, 2020.

[19] T. Hong, X. Li, Z. Zhu, and Q. Li, "Optimized structured sparse sensing matrices for compressive sensing," Signal processing, vol. 159, pp. 119-129, 2019.

[20] J. Guo and R. Wang, "Alternating optimisation seeking generates elemental amplitudes combined with chaotic random phases to construct a circular measurement matrix," Acta Physic Sinica, vol. 64, no. 13, pp. 55-66, 2015.

[21] X. Kong, H. Bi, D. Lu, and N. Li, "Construction of a class of logistic chaotic measurement matrices for compressed sensing," Pattern Recognition and Image Analysis, vol. 29, no. 3, pp. 493-502, 2019.

[22] S. Zhang, D. Wang, and Y. Yan, "Instrumental variable-based OMP identification algorithm for Hammerstein systems," Complexity, vol. 2018, Article ID 8420426, 10 pages, 2018. 